\title{
EchoGéo
}

$10 \mid 2009$

La piraterie

\section{La diaspora, instrument de la politique de puissance et de rayonnement de l'Inde à l'île Maurice et dans le monde}

Anouck Carsignol-Singh

\section{OpenEdition}

Journals

Édition électronique

URL : https://journals.openedition.org/echogeo/11329

DOI : 10.4000/echogeo.11329

ISSN : 1963-1197

Éditeur

Pôle de recherche pour l'organisation et la diffusion de l'information géographique (CNRS UMR 8586)

Référence électronique

Anouck Carsignol-Singh, « La diaspora, instrument de la politique de puissance et de rayonnement de I'Inde à l'île Maurice et dans le monde », EchoGéo [En ligne], 10 | 2009, mis en ligne le 26 novembre 2009, consulté le 16 septembre 2021. URL : http://journals.openedition.org/echogeo/11329 ; DOI : https://doi.org/10.4000/echogeo.11329

Ce document a été généré automatiquement le 16 septembre 2021.

EchoGéo est mis à disposition selon les termes de la licence Creative Commons Attribution - Pas d'Utilisation Commerciale - Pas de Modification 4.0 International (CC BY-NC-ND) 


\title{
La diaspora, instrument de la politique de puissance et de rayonnement de l'Inde à l'île Maurice et dans le monde
}

\author{
Anouck Carsignol-Singh
}

\section{Introduction}

1 L'utilisation par l'Inde des instruments classiques de la puissance étatique tels que la force nucléaire ou la pression économique ne doit pas occulter l'importance de la politique de Soft Power ${ }^{1}$, adoptée dès le début du $\mathrm{XX}^{\text {th }}$ siècle par les leaders nationalistes indiens et qui caractérise la politique étrangère du pays aujourd'hui encore. La diaspora, principal instrument du rayonnement culturel et politique de l'Inde à l'étranger au cours de la première moitié $\mathrm{du} \mathrm{XX}^{\mathrm{e}}$ siècle, a été délaissée au lendemain de l'Indépendance par le gouvernement et la société indienne post-coloniale et n'a été réhabilitée que récemment. Aujourd'hui, l'Inde s'appuie sur une diplomatie culturelle et ethnique à la fois idéaliste et pragmatique à l'égard des Indiens d'outremer, pour rehausser l'image du pays et défendre ses intérêts aux échelles locale, régionale et internationale.

2 L'importance des immigrés indiens, les Non Resident Indians (NRI) ${ }^{2}$, est aujourd'hui reconnue ouvertement par New Delhi qui en fait l'un des piliers de sa politique étrangère. Celle des Persons of Indian Origin (PIO) ${ }^{3}$ est moins évidente, malgré le rôle de la diaspora dans la politique d'affirmation et de consolidation de la puissance émergente de l'Inde.

3 Seul pays au monde dont la population est majoritairement composée de PIO (71\%), l'île Maurice a également toujours été gouvernée par des leaders d'origine indienne depuis l'Indépendance en 1968 - à l'exception du court mandat de Paul Bérenger, Premier ministre de 2003 à 2005. L'Inde et Maurice entretiennent certes des relations 
privilégiées, mais les Indo-Mauriciens ne se contentent pas de promouvoir les intérêts de leur pays d'origine à Maurice ; leur statut dans l'île étant étroitement lié à l'image de l'Inde sur la scène internationale, les PIO s'investissent également dans le but de consolider leur propre position au niveau local. Mus par des facteurs aussi bien endogènes qu'exogènes, ils se mobilisent pour défendre à la fois les valeurs morales et culturelles et les intérêts géostratégiques et politiques de l'Inde, aux échelles régionale et globale. Les PIO de l'île Maurice offrent ainsi un cas d'étude singulier sur le rôle du facteur diasporique dans la consolidation de la puissance étatique de l'Inde sur la scène internationale, par des moyens non-conventionnels.

\section{Les relations tourmentées entre l'Inde et sa diaspora}

4 A l'heure du rapprochement inédit entre New Delhi et sa diaspora ${ }^{4}$, la relation historique entre l'Inde et ses émigrés reste méconnue, et le poids de la diaspora dans la politique étrangère du pays, sous-estimé. Plurielle, la diaspora indienne entretient avec New Delhi une relation à géométrie variable, particulièrement sensible aux facteurs spatio-temporels, que l'on peut découper chronologiquement en trois phases: l'instrumentalisation des PIO par les mouvements nationalistes indiens au cours de la première moitié du $\mathrm{XX}^{\mathrm{e}}$ siècle, la dissociation active du gouvernement indien à l'égard des émigrés sous l'ère nehruvienne, puis la réconciliation à partir des années 1990, qui se traduit par la mise des NRI au service de la Realpolitik de New Delhi, tandis que les PIO deviennent l'instrument privilégié du Soft Power de l'Inde dans le monde.

\section{La naissance d'une diaspora plurielle}

5 Si la mobilité des Indiens s'inscrit dans le cadre d'une tradition migratoire ancienne, dans et hors du sous-continent, la mise en place du système de l'engagisme par la Grande-Bretagne à partir de 1834 inaugure un mouvement massif et définitif de population, à l'origine de la naissance d'une diaspora indienne laborieuse. Plus de 1,5 million d'Indiens ont quitté le sous-continent entre 1834 et 1917 et ont été envoyés vers Maurice (450 000), les Caraïbes (200 000), la Guyane britannique (239 000), le Suriname (34 300), les îles Fidji (61 000), mais aussi en Afrique de l'Est (32 000) et du Sud (Natal, 152 000) (Tinker, 1974). En Asie du Sud-Est, près de six millions de travailleurs indiens ont été exportés en direction des pays voisins dans le cadre d'un système similaire, appelé maystri au Myanmar (2,5 millions) et kangani dans la péninsule malaise (2 millions) et à Ceylan (Ministry of External Affairs, 2002). A la suite des Anglais, les Français et les Hollandais ont également eu recours à l'engagisme, à l'origine de la présence d'Indiens en Insulinde, à l'île Bourbon et dans les Antilles françaises (Guilmoto, 1991).

6 Les travailleurs d'origine indienne, recrutés dans les plantations pour remplacer les esclaves nouvellement affranchis, sont rejoints par des migrants libres qui forment une classe marchande et une petite élite intellectuelle, et qui conservent des liens non seulement commerciaux, mais aussi culturels et politiques avec le sous-continent. Passagers libres et engagés du XIX ${ }^{\mathrm{e}}$ siècle sont aujourd'hui considérés par l'Inde comme des PIO, et constituent la majorité de la diaspora indienne dans le monde. 
7 Au lendemain de l'Indépendance de l'Inde et de la création de nouveaux États en Asie du Sud, deux nouveaux mouvements migratoires prennent forme, stimulés par la demande de main-d'œuvre qualifiée dans les pays occidentaux, et le besoin d'ouvriers peu ou non qualifiés au Moyen-Orient. Suite à la vague d'émigration élitiste en direction des pays développés, on recense à ce jour environ 2,7 millions d'Indiens aux États-Unis ${ }^{5}, 1,2$ million au Royaume Uni ${ }^{6}$ et à peu près autant au Canada ${ }^{7}$. Toujours détenteurs de leur nationalité indienne, ces émigrés constituent la diaspora des NRI, et sont érigés en véritable « minorité modèle » aux États-Unis.

8 Dans les pays du Golfe, plus de 4 millions d'Indiens sont employés sur la base de contrats temporaires et précaires (Venier, 2003 ; Percot, 2005). La plupart (750 000) sont installés dans les Emirats Arabes Unis, où ils constituent $32 \%$ de la population totale. Ils sont 150000 à Bahreïn (20\%), 200000 au Koweït (13\%), 450000 en Oman (15\%), 100000 au Qatar (24\%), 1200000 en Arabie Saoudite (7\%) et 130000 dans les autres pays du Golfe (Ministry of External Affairs, 2002). Alors que le premier mouvement a longtemps été décrit comme une "fuite des cerveaux » des pays du Tiers Monde en direction de pays développés, le second apparaît comme un véritable Coolie trade ${ }^{8}$ des temps modernes.

\section{La diaspora des PIO, levier du mouvement nationaliste en Inde et à l'extérieur}

9 L'Inde du début du $\mathrm{XX}^{\mathrm{e}}$ siècle est secouée par l'émergence de mouvements nationalistes. Ceux-ci tentent de valoriser les liens entre le sous-continent et ses anciens ressortissants, pour présenter leur pays non pas comme une colonie assujettie, mais comme une grande puissance coloniale. Après avoir d'abord dénigré les Coolies, les nationalistes indiens entreprennent de les élever au rang de " colons ", « ambassadeurs de la culture indienne ", « constructeurs d'empire », " pionniers » ou " aventuriers " Cette transformation discursive du statut de l'immigrant vise à rehausser le statut de l'Inde dans l'Empire britannique et sur la scène internationale. C'est dans ce contexte que deux organisations nationalistes indiennes, la Greater India Society et l'Indian Colonial Society, prennent l'initiative de commémorer le centenaire de ce qu'ils appellent la « colonisation indienne » à Maurice. Cette cérémonie, organisée en 1935 à Port Louis (île Maurice), marque une véritable instrumentalisation de la diaspora par l'intelligentsia indienne, qui défend le projet expansionniste et universaliste d'une Greater India, non pas tant pour soutenir les velléités autonomistes en Inde que pour valoriser la place des émigrés indiens dans la société insulaire, à une époque où la majorité des Indo-Mauriciens sont encore considérés et se perçoivent eux-mêmes comme des Coolies. Cette commémoration constitue également la première occasion pour les Indo-Mauriciens de se rassembler en masse, en face du Champ de Mars, lieu associé jusque-là aux « courses malabars $»^{10}$. A quelques mètres du champ de courses, un obélisque (photo 1) est inauguré par l'invité d'honneur, le militant nationaliste indien T. K. Swaminathan, transformant ce site d'humiliation historique en un lieu de triomphe de l'indianité à Maurice. 
Photos 1 \& 2 - L'obélisque du Champ de Mars, Port Louis, île Maurice
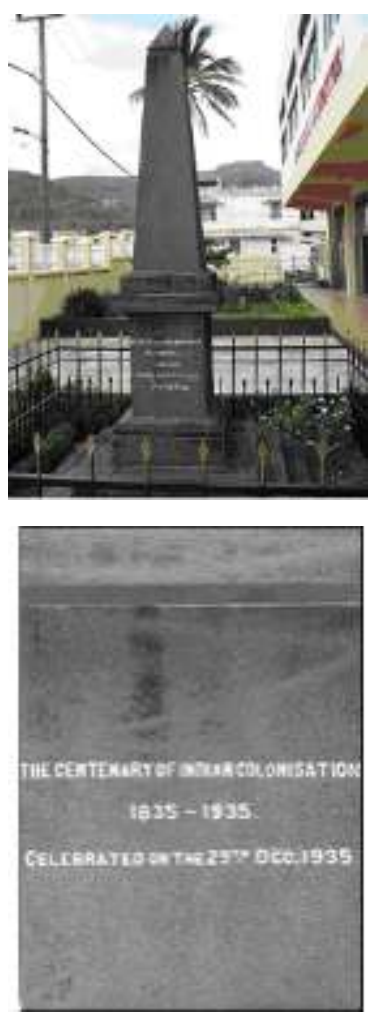

Clichés de l'auteur

10 L'inscription gravée sur l'obélisque (Photo 2) est transcrite en quatre langues : anglais, hindi, ourdou et tamoul, symbole de la diversité et de l'unité de la communauté indomauricienne. La célébration marque le début d'une prise de conscience identitaire et d'une mobilisation politique des Indo-Mauriciens et est à l'origine de la montée en puissance des Biharis à Maurice. L'année 1935 célèbre en effet le centenaire non pas du début de l'immigration indienne en général, mais de l'engagisme, c'est-à-dire l'arrivée massive de travailleurs en majorité hindous du Nord de l'Inde (originaire des États actuels du Bihar et de l'Uttar Pradesh). Le choix de cette date laisse donc de côté les Tamouls et les musulmans d'origine indienne, débarqués dans l'île dès le XVII ${ }^{e}$ siècle. En initiant cette commémoration, les mouvements nationalistes indiens contribuent à ethniciser le récit historique ainsi que les relations inter-communautaires au sein de la communauté indo-mauricienne, et propulsent l'élite hindoue sur le devant de la scène socio-politique insulaire, posant ainsi les fondations de l'hégémonie politique et culturelle des Biharis sur les autres communautés à Maurice ${ }^{11}$.

11 L'organisation conjointe du centenaire de la colonisation par l'élite indo-mauricienne et les leaders nationalistes indiens illustre enfin l'instrumentalisation réciproque des mobilisations identitaires en Inde et à Maurice en vue de répondre à des enjeux locaux croisés, comme le résume M. C. Pillay, membre de l'Indian Cultural Association:

"The significance of the Centenaries festivities is too obvious to be mistaken. On the one hand, through the participation of Mr Swaminathan as the delegate of the Indian Colonial Society they signify India's desire to establish a link between the Colonies and herself, and on the other they emphasize the importance of the work done by Indians in this island which we cherish as our home.» (PILLAY M. C., 'Introductory notes', Indian Centenary Book, 10-02-1936) 
12 La première moitié du $\mathrm{XX}^{\mathrm{e}}$ siècle est ainsi marquée par une convergence d'intérêts entre, d'une part, l'intelligentsia nationaliste en Inde, et, d'autre part, des leaders d'origine indienne en diaspora, dans le cadre d'une lutte commune contre l'administration coloniale britannique.

\section{La phase de dissociation active entre l'Inde et sa diaspora}

13 En 1947, alors que le gouvernement de l'Inde post-coloniale doit avant tout asseoir sa légitimité au niveau interne, consolider ses frontières vis-à-vis de ses voisins et affirmer sa place sur la scène internationale, la diaspora indienne est reléguée aux marges de la politique nehruvienne. L'internationalisme du début du siècle est remplacé par un tiers-mondisme solidaire des nationalismes émergents. Pour Sunil Khilnani, 'Nehru believed that an Indian identity could emerge only within the territorial and institutional form of a state' (Khilnani, 1997, 167).

Les discours de certains nationalistes, certes marginaux, relatifs à la Greater India et au pan-asianisme, inquiètent par ailleurs les gouvernements des pays asiatiques nouvellement décolonisés, à l'instar de Myanmar, de Ceylan et de la Malaisie, qui y voient un risque d'intrusion et craignent que l'impérialisme britannique ne soit remplacé par une domination indienne. Au cours de l'Asian Relations Conference de 1947, alors que Nehru ré-affirme les liens culturels et spirituels qui unissent les Indiens de Birmanie à leur pays ancestral, il reçoit de son homologue birman une note l'exhortant à ne plus revendiquer publiquement l'appartenance à l'Inde des émigrés installés au pays. Les pressions ainsi exercées sur l'Inde par les États récemment décolonisés incitent le gouvernement du Congrès à prendre ses distances à l'égard des Indiens d'outremer.

Les problèmes liés à la diaspora indienne, qui mettent directement en cause la souveraineté des pays d'installation, ne peuvent plus être renvoyés à la responsabilité impériale de la Grande-Bretagne. Or l'Inde nouvellement indépendante ne peut intervenir en faveur des Indiens d'outremer sans mettre en péril ses relations diplomatiques inter-étatiques ainsi que sa crédibilité internationale. Dès lors, New Delhi se méfie des communautés indiennes d'Afrique ou d'Asie, perçues comme un obstacle à la Moralpolitik de l'Inde, et comme un frein aux mouvements anti-coloniaux. La politique nehruvienne à l'égard des PIO peut se résumer par cette déclaration, prononcée par le Premier Ministre à la veille de la conférence de Bandung :

«Je pense que les Indiens d'Afrique ont fait beaucoup de choses. Certains ont également réalisé beaucoup de profit. Je pense que les Indiens d'Afrique, ou d'ailleurs, peuvent être utiles pour la communauté. Mais nous n'apprécions leur rôle que sur cette base : nous devons accorder la priorité aux intérêts des peuples d'Afrique $»^{12}$.

Pour Bikhu Parekh, 'The Indian lack of interest in overseas Indians had also its roots in its patronising attitude to them. For the politically minded Indians, including Nehru and the socialists, overseas Indians were either poor or illiterate and this was a liability, or they were rich men who exploited the local population and thereby an embarrassment' (Parekh, 1993, 10). Nehru recommande dès lors aux émigrés indiens de s'intégrer dans leur société d'installation, dans le double but de favoriser les relations inter-communautaires locales et faciliter leur sédentarisation d'une part, et de dé-responsabiliser l'Inde vis-à- 
vis des émigrés d'autre part. Les préoccupations du parti du Congrès au lendemain de l'Indépendance traduisent ainsi un rétrécissement du champ d'action du gouvernement, de la scène transnationale aux relations stato-centrées. Partisan d'une «non-intervention attentive », Nehru limite les relations entre l'Inde et les Indiens d'outremer au domaine culturel (Benoist, 1988). Dans la continuité de la politique de son père, Indira Gandhi garde ses distances par rapport à la diaspora et s'oppose notamment à l'entrée en Inde des PIO originaires du Kenya et d'Ouganda, expulsés de ces pays au cours des années 1970 .

\section{La réconciliation: les NRI mis au service de l'économie indienne}

17 Le rapprochement entre l'Inde et la diaspora est principalement dû aux deux nouvelles vagues migratoires qui se déroulent au cours des années 1960-1970. La première, hautement qualifiée, installée principalement en Amérique du Nord, constitue un potentiel économique inexploité pour l'Inde. La seconde, laborieuse, recrutée dans les monarchies pétrolières du Moyen-Orient, est extrêmement généreuse en envois de fonds ${ }^{13}$. A partir de 1984, le Premier Ministre Rajiv Gandhi est le premier à prendre conscience de l'importance stratégique des NRI, qu'il considère non pas comme une élite en fuite, mais comme une " banque de cerveaux » :

«Nous faisons un blocage sur l'exode des cerveaux. Nous ne devrions pas nous en inquiéter (...). Nous de devons pas considérer ce phénomène comme une perte. Nous ne devons pas l'envisager comme un exode des cerveaux, mais comme une banque de cerveaux qui accumule des intérêts et qui attend uniquement que nous le retirions et que nous l'investissions à nouveau en Inde » (Gandhi, 1987, 214)

Progressivement, les Indiens d'outremer bénéficient de nouveaux droits fiscaux, d'une reconnaissante croissante de la part du gouvernement, et d'une participation économique et politique accrue.

La réconciliation entre État et NRI est confortée en 1998 par l'arrivée au pouvoir du Bharatiya Janata Party (BJP, considéré comme le parti de la droite hindoue), qui renforce la politique d'institutionnalisation de la diaspora. Le High Level Committee on the Indian Diaspora $^{14}$ jette les bases de la nouvelle politique de New Delhi à l'égard de la frange privilégiée de la population indienne d'outre mer. Suivant les recommandations du rapport rendu par ce Haut Comité en 2002, le gouvernement nomme un Ambassador-atlarge for NRI and PIO, met en place un ministère consacré aux affaires des Indiens d'outremer (Ministry of Overseas Indians Affairs, MOIA) et cherche à réhabiliter l'image des NRI en Inde. En 2003 a ainsi lieu le premier Pravasi Bharatiya Divas, cérémonie annuelle en l'honneur de la diaspora, dont le point d'orgue consiste en la remise du Pravasi Bharatiya Saman récompensant les contributions des émigrés Indiens au développement de l'Inde ou au bien-être de la communauté d'origine indienne à l'étranger. De nombreuses autres mesures ont été adoptées par le gouvernement dominé par le parti du Congrès qui accède au pouvoir en 2004. Ainsi, les NRI peuvent désormais bénéficier du statut d'Overseas Citizen of India (OCI), qui leur donne accès à la double nationalité (à l'exception de l'exercice de droits civiques et de l'acquisition de propriété agricole). Célébrés en véritables fils prodigues, les NRI sont invités à mettre leurs ressources financières ou intellectuelles au bénéfice de l'Inde. 


\section{Les PIO, relais de la présence culturelle de l'Inde à I'île Maurice}

S'il est vrai que les NRI constituent l'une des nouvelles priorités de la politique de l'Inde, les PIO jouent un rôle essentiel dans la promotion de la culture et de la politique indienne dans leur pays d'installation. A l'île Maurice, le gouvernement central et les différents États de l'Union indienne cultivent les liens historiques et sentimentaux des Indo-Mauriciens envers l'Inde, en finançant les centres culturels hindou, tamoul, télougou, marathi, en encourageant la pratique du 'roots tracing' 15 , ou en parrainant des festivals et autres cérémonies indiennes dans l'île. Deux exemples illustrent l'importance attachée par les uns et les autres à leurs origines communes : il s'agit de la promotion de Grand Bassin et de l'Aapravasi Ghat au rang de sites historiques et mythiques de renommée internationale. L'intervention de l'Inde dans la gestion du patrimoine national mauricien se fait certes au nom d'affiliations ethno-culturelles et de valeurs morales communes, mais aussi à des fins stratégiques, dans la perspective de consolider les relations bilatérales Inde-Maurice, et d'améliorer le statut des deux pays.

Carte de l'île Maurice

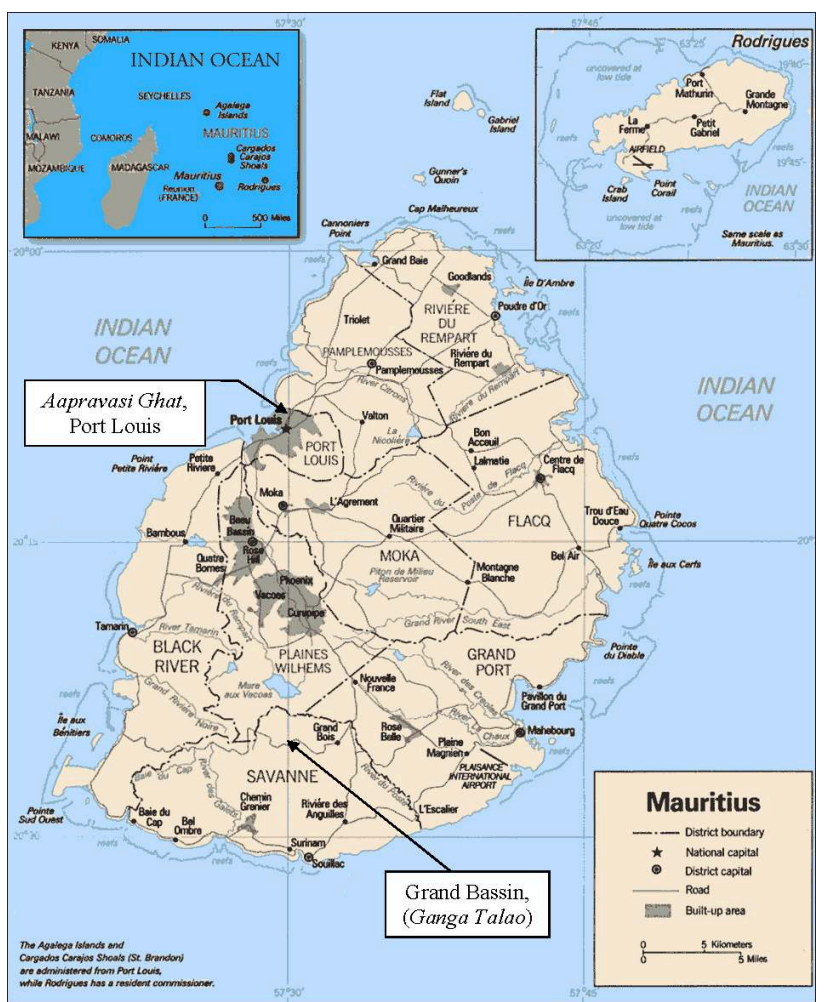

\section{Ganga Talao : I'hindouisation du patrimoine naturel mauricien}

Objet de légendes et de mythes, le lac volcanique Grand Bassin, situé au centre de l'île, a nourri l'imaginaire des esclaves malgaches du XVIII ${ }^{\mathrm{e}}$ siècle, qui y voyaient une échappatoire vers leur île natale. Au XIX ${ }^{\mathrm{e}}$ siècle, les engagés indiens élaborent à leur 
tour des légendes autour du lac, également perçu comme un lien virtuel vers leur pays natal. Consacré en rêve par le pandit (prêtre hindou) Jhummun Giri Gossagne en 1890, Grand Bassin est devenu un lieu mythique dans l'imaginaire collectif indo-mauricien, avant d'être transformé en 'tirtha', site de pèlerinage populaire, à partir de 1898. Dans les années 1950, le lac mystique fait l'objet d'une appropriation de la part des leaders communautaires hindous, soucieux de protéger le site, mais aussi de l'utiliser comme symbole identitaire, source de légitimité culturelle et trait d'union virtuel entre l'île Maurice et l'Inde.

Situé au cœur d'une zone forestière, Grand Bassin (Photo 3) a longtemps été fréquenté par les chasseurs, avant d'être soustrait à la juridiction du département des Forêts en 1962 et placé sous la tutelle de l'association culturelle Hindu Maha Sabha pour aménager le site en lieu de pèlerinage. Le 21 juin 1972, lors d'une cérémonie publique conjointement organisée et financée par les gouvernements indien et mauricien, le pandit indien Vidhya Nidhi Pandey est invité à Maurice pour verser l'eau du Gange dans le lac, officialisant ainsi un lien physique et symbolique entre Ganga Talao et le fleuve sacré indien. Depuis, nombre de leaders religieux et politiques, d'artistes et de personnalités indiennes ont contribué à l'hindouisation du site, à l'instar du ministre des Affaires étrangères de l'Inde, Atul Bihari Vajpayee, qui lors de sa visite en 1977 a suggéré la construction d'un temple d'Hanuman, en partie financé par le gouvernement indien et le Sanatan Sabha de Delhi. Durant son passage à Maurice, le chanteur indien Gulshan Kumar a, quant à lui, proposé l'érection d'une statue de Shiva sur la rive de Ganga Talao. Symbole d'une visibilité accrue de l'indianité et en particulier de l'hindouisme à Maurice, elle a été réalisée par le sculpteur indien Matu Ram Verma, et inaugurée en 2007. Haute de 108 pieds (33 mètres) ${ }^{16}$, c'est la plus grande statue de Shiva au monde (Photo 4).

Photos 3 et 4 - La statue de Shiva, sur la rive de Grand Bassin, île Maurice, 2008

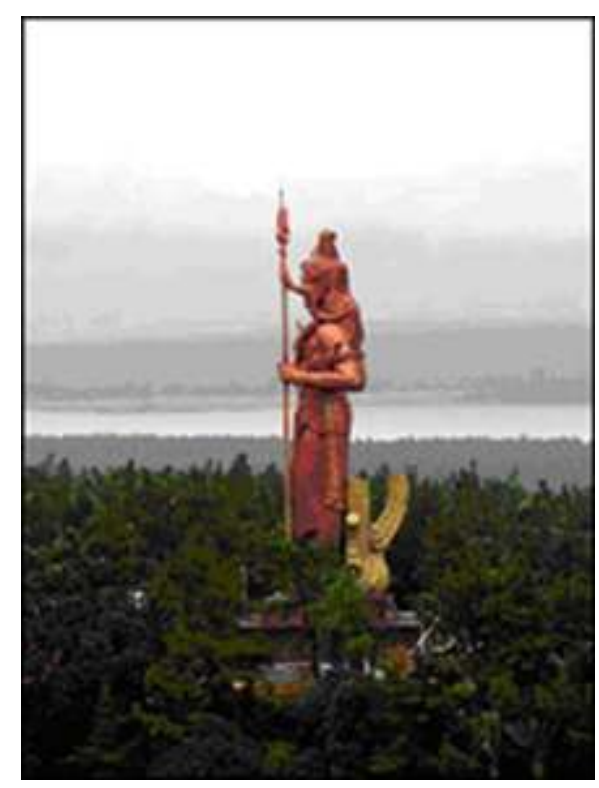

Source photo 3 : http://cariland.unblog.fr/2008/03/04/maurice-un-paradis 


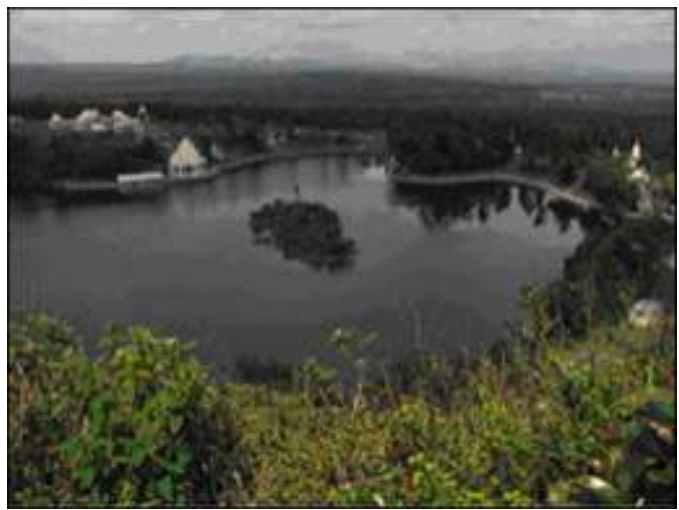

Photo 4 : cliché de l'auteur.

La sacralisation officielle de Grand Bassin marque non seulement l'appropriation conjointe du site par les leaders politiques hindous de Maurice et d'Inde, mais fait également de l'Inde un co-producteur de sens dans l'île, en association avec la diaspora et le gouvernement insulaire. Chaque année, Ganga Talao voit affluer pèlerins et visiteurs, en particulier à l'occasion du Maha Shivaratree (fête majeure du calendrier hindou, au mois de février ou mars) qui attire plus de 300000 personnes. Site de pèlerinage national, Ganga Talao a acquis une renommée régionale, voire internationale, et de nombreux dévots se déplacent d'Afrique du Sud, de Singapour, de Malaisie et même de l'Inde, pour prendre part à la célébration. La transformation de Grand Bassin en Ganga Talao illustre le rôle de l'Inde dans la valorisation des IndoMauriciens dans le paysage insulaire, en particulier des hindous. En faisant de Maurice une 'petite Inde' ou chota Bharat (Hookoomsing, 2003), New Delhi renforce également sa présence et son influence culturelle dans la région.

\section{L'Aapravasi Ghat : un trait d'union entre l'île Maurice et I'Inde}

Autre exemple d'appropriation du patrimoine mauricien par la diaspora indienne, avec le soutien de New Delhi, l'Aapravasi Ghat (quai des immigrants, Photo 5) fait l'objet de l'attention particulière des gouvernements indien et mauricien, qui se servent de ce vestige de l'engagisme pour renforcer leurs liens, et accroître leur prestige international à travers une stratégie de victimisation-valorisation. Pendant presque un siècle, les travailleurs engagés et les esclaves affranchis ont transité par le 'Dépôt d'immigration' à leur arrivée à Maurice. Etant donné la forte proportion d'Indiens parmi les immigrants, le débarcadère est connu sous le nom de Coolie Ghat (quai des Coolies). A la fin de l'engagisme, les bâtiments du Dépôt cristallisent aux yeux des engagés et de leurs descendants le souvenir d'un voyage traumatisant et l'humiliation du travail semi-féodal dans les plantations; ils sont laissés à l'abandon ou réaffectés à d'autres usages. Ce n'est qu'au lendemain de l'Indépendance, en 1968, que l'élite indomauricienne prête un intérêt nouveau au site : l'engagisme appartient alors au passé et sa commémoration ne risque plus de freiner l'ascension socio-économique et politique des Mauriciens d'origine indienne ; au contraire il leur sert de faire-valoir vis-à-vis des autres communautés. 

Gandhi, le Dépôt des immigrants fait l'objet d'une appropriation par la communauté indo-mauricienne, et en particulier par les Biharis de confession hindoue. Le 2 novembre 1970 , le journaliste B. Ramlallah organise pour la première fois un yaj (cérémonie religieuse) en mémoire de "l'esprit fraternel des engagés» (jehaji bhai). Malgré sa vocation initiale visant à célébrer l'unité des Indo-Mauriciens, cette prière, devenue annuelle, contribue à associer étroitement l'expérience de l'engagisme, le Dépôt des immigrants et la communauté hindoue dans la perception collective.

L'appropriation populaire et symbolique du Coolie Ghat est, par la suite, officialisée par le gouvernement mauricien: en 1985, le Coolie Ghat devient monument protégé par l'État et deux ans plus tard, le site est officiellement re-baptisé Aapravasi Ghat. Cette transformation taxonomique en faveur d'un terme d'origine sanskrite vise à re-hausser le statut des Indo-Mauriciens, qui se définissent non plus comme les descendants des Coolies mais comme des aapravasi (immigrants). Il constitue également un lieu d'exclusion pour les non-hindiphones. Ainsi, l'iconographie et la terminologie en hindi ou sanskrit réduisent les musulmans, Tamouls, Télougous et Marathis à un rôle secondaire dans l'ethno-histoire de la communauté indo-mauricienne, et effacent les Chinois et Africains du récit historique de l'engagisme. Décrété 'patrimoine national' en 2003, l'Aapravasi Ghat est progressivement transformé en un lieu public de commémoration nationale, auquel seuls s'identifient les Indo-Mauriciens de confession hindoue et de langue maternelle hindi ou bhojpuri (Photo 6).

Photos 5 \& 6 - Les 14 marches de l'Aapravasi Ghat et le Monument de commémoration des l'arrivée des engagés indiens
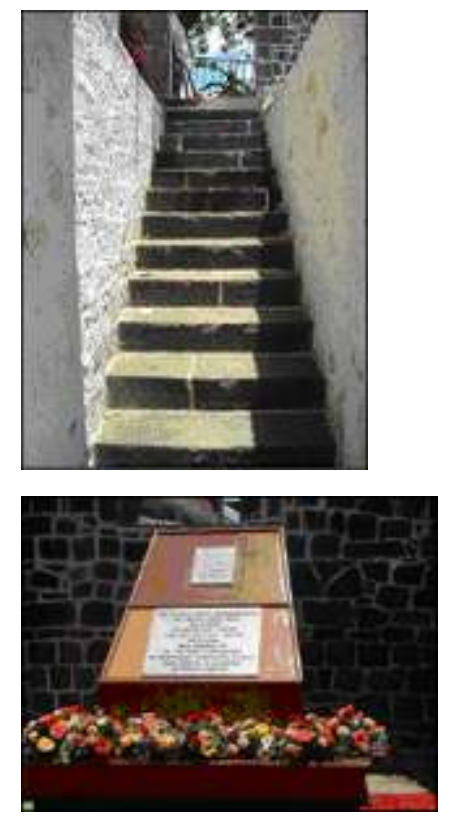

Port Louis, île Maurice, 2 novembre 2005 ; Clichés de l'auteur

En 2001, un décret du gouvernement renforce le processus d'ethnicisation du patrimoine mauricien en proclamant deux journées nationales dédiées à la commémoration de l'arrivée des premiers engagés et à la célébration de l'abolition de l'esclavage: le 2 novembre, associé à l'ethno-histoire des Indo-Mauriciens et le $1^{\mathrm{er}}$ février considéré comme le jour des Créoles $^{17}$. Passant outre les différences 
fondamentales entre l'esclavage et l'engagisme, les leaders indo-mauriciens évoquent ces deux pans de l'histoire mauricienne sur un pied d'égalité et estiment que les engagés auraient subi des traumatismes comparables à ceux endurés par les esclaves. La comparaison entre esclavage et engagisme est poussée à son paroxysme en 1998 lorsque Dhundev Bahadoor, Président de GOPIO Mauritius (Global Organisation of People of Indian Origin) et du Human Service Trust, l'un des représentants les plus influents de la communauté hindoue à Maurice, s'est mobilisé pour que les descendants des engagés obtiennent de la Grande-Bretagne une indemnisation financière de dédommagement, au même titre que les descendants des esclaves. Au projet de 'route des esclaves' proposé par l'UNESCO en 1994, répond celui de la 'route du Coolie', porté par des entrepreneurs identitaires indo-mauriciens et soutenu par le Premier ministre Navin Ramgoolam.

Par ailleurs chaque 2 novembre, les leaders politiques, en majorité PIO, font de l'Aapravasi Ghat le symbole de l'ascension socio-politique exceptionnelle des IndoMauriciens, et en particulier des hindous à Maurice, se félicitant au passage de leur propre trajectoire personnelle en tant que descendants d'engagés. Le Premier ministre actuel, Navin Ramgoolam, se plaît ainsi à raconter une anecdote familiale selon laquelle son père eut un jour à subir les propos méprisants d'un notable : 'Mon grand-père était un avocat, mon père était un avocat, je suis un avocat', ce à quoi Seewoosagur Ramgoolam lui aurait rétorqué : 'Mon grand-père était coolie, mon père était coolie et je suis médecin'18. A l'occasion de la cérémonie du 2 novembre, une représentation théâtrale met en scène et dramatise les difficiles conditions de vie et de travail des travailleurs indiens (Photo 7).

Photo 7- Représentation théâtrale de l'arrivées des engagés, Aapravasi Ghat

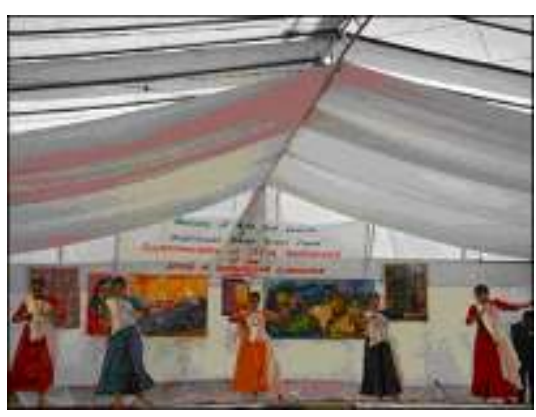

Port Louis, 2 novembre 2005 ; Cliché de l'auteur

'engagisme, qui a longtemps constitué une source d'humiliation et de stigmatisation, est ainsi transformé de manière discursive en une ressource symbolique ${ }^{19}$, et constitue aujourd'hui l'occasion pour la communauté hindoue d'afficher sa réussite et de consolider son influence dans l'île, au risque d'accréditer la thèse de la "communauté modèle » au détriment des autres groupes en présence ${ }^{20}$.

Cette politique de reconnaissance permet de resserrer les liens historiques entre l'Inde et l'île. Depuis 1970, la visite du site est d'ailleurs systématiquement inscrite à l'agenda des représentants officiels indiens de passage ${ }^{21}$. Le gouvernement indien et les organisations socio-culturelles et religieuses indiennes ont également apporté un appui financier et technique au projet de réhabilitation de l'Aapravasi Ghat, et donnent aujourd'hui au dépôt une importance symbolique et une visibilité internationale. Lors des éditions 2006 et 2007 des Pravasi Bharatiya Divas en Inde, un atelier animé par la 
délégation mauricienne a été consacré à l'Aapravasi Ghat. Dans la perspective de bénéficier de la nouvelle publicité dont fait l'objet l'engagisme dans les institutions internationales, le ministère des Affaires des Indiens d'outremer a même initié le projet de réhabiliter le Dépôt d'émigration de Bhowanipore à Kolkata. Par ailleurs, chaque 2 novembre, la présence d'une délégation officielle indienne à Maurice contribue à ancrer l'idée d'un lien exclusif entre l'engagisme et les Indo-Mauriciens dans la perception collective, non seulement des Mauriciens, mais également de l'opinion publique internationale.

Depuis 2006, l'Aapravasi Ghat est inscrit au patrimoine mondial de l'UNESCO, en tant que dernier dépôt d'immigrants engagés au monde. A ce titre, l'ICOMOS (International Council of Monuments and Sites, une organisation internationale non-gouvernementale qui coopère avec l'UNESCO en matière de préservation du patrimoine historique mondial) a rappelé au gouvernement mauricien le caractère universel de l'engagisme et lui a demandé expressément de dés-ethniciser le site de l'Aapravasi Ghat pour permettre aux membres des communautés non-indiennes de s'y identifier à leur tour.

Le soutien de l'Inde au projet de réhabilitation du Dépôt des immigrants à Maurice s'inscrit non seulement dans le cadre d'une politique de protection du patrimoine historique commun aux deux pays, mais s'apparente également à une entreprise de valorisation de la communauté indienne dans l'île. Les Indo-Mauriciens, et en particulier le groupe majoritaire, composé d'hindous d'origine biharie, voient ainsi leur prééminence socio-culturelle et politique consolidée par l'appui extérieur de $\mathrm{New}$ Delhi, dans le cadre d'une relation Inde-Maurice aux intérêts croisés.

\section{La diaspora, instrument de smart power de l'Inde dans le monde}

Dans la continuité de l'idéalisme pratique' prôné par Nehru, les gouvernements successifs ont combiné la défense des idéaux universalistes de l'Inde et la protection des intérêts pragmatiques du pays dans le cadre d'une politique étrangère mixte. Depuis les années 1990, l'Inde s'appuie sur sa diaspora non seulement à des fins de développement national ou dans le cadre de relations bilatérales, mais aussi à l'échelle régionale et internationale. La mobilisation de la diaspora par New Delhi s'inscrit dans la nouvelle politique de puissance, qui met la Moralpolitik et la diplomatie culturelle au service de la Realpolitik de l'Inde.

\section{La diaspora et l'État indien : convergence d'intérêts et valorisation réciproque}

34 A partir des années 1990, le gouvernement indien encourage la création de nombreux organismes diasporiques, afin de promouvoir les intérêts de l'Inde auprès des institutions gouvernementales de leur pays d'installation et d'exercer un lobbying actif en faveur de la politique extérieure de New Delhi ${ }^{22}$. Ainsi la Global Organization of People of Indian Origin (GOPIO), principale organisation diasporique indienne à dimension globale, basée à New York, se rallie à la quasi totalité des projets du gouvernement indien et agit dans ses pays d'implantation comme une véritable ambassade informelle de l'Inde. L'une des branches de la GOPIO, implantée à Maurice et représentative de la 
diaspora des PIO, est dotée d'une forte capacité d'influence auprès du gouvernement mauricien, dont certains des membres les plus éminents figurent dans l'organigramme de l'association, tels Raouf Bundhun, vice-Président de l'île Maurice de 2002 à 2007, ou Navin Ramgoolam, actuel Premier ministre de Maurice, nommé 'patron' de la GOPIO Mauritius. Inversement, les dirigeants de la GOPIO Mauritius sont particulièrement bien introduits au gouvernement mauricien, tels l'ancien président, Dhundev Bauhadoor, l'un des plus influents lobbyistes de l'île, ou de son successeur, Mahendra Utchanah, qui bénéficie de contacts hauts placés en tant qu'ancien ministre de l'Energie à Maurice. Fidèle à la politique de New Delhi, la GOPIO a notamment affiché son soutien aux essais nucléaires indiens de 1998, entrepris par le gouvernement du BJP en violation du Traité de Non-Prolifération des armes nucléaires de 1968, dont l'Inde n'est pas signataire.

Le gouvernement mauricien et la communauté indo-mauricienne s'expriment d'une seule et même voix lorsqu'il s'agit de soutenir la politique de puissance de New Delhi. Cette convergence est principalement due au fait que l'État mauricien et la diaspora indo-mauricienne se définissent tous deux par rapport à l'Inde, et ont chacun un intérêt propre à promouvoir sa place sur la scène internationale. Tandis que le soutien affiché à l'Inde apporte à la diaspora un statut prestigieux à l'île Maurice et lui permet de renforcer sa prééminence socio-culturelle et politique sur les autres communautés insulaires, l'État mauricien y gagne quant à lui un partenaire économique, politique et stratégique indispensable au développement du pays.

\section{Lîle Maurice, tête de pont de l'Inde dans l'Océan Indien}

En 1970, deux ans à peine après l'Indépendance de l'île, Indira Gandhi est le premier chef d'État étranger à se rendre à Port-Louis en visite officielle, inaugurant ainsi des relations diplomatiques privilégiées entre l'Inde et Maurice. Depuis, l'Inde a accueilli 14 fois le Premier ministre et 6 fois le Président de la République de Maurice en visite officielle, tandis qu'à Maurice ont eu lieu 9 visites officielles de Premiers ministres indiens et 3 visites présidentielles. Lors de son déplacement au Punjab en décembre 2008, le Président mauricien Anerood Jugnauth est allé jusqu'à évoquer les liens de sang qui unissent les deux pays: "Indo-Mauritius ties go beyond friendship; we share blood relation with each other because the forefathers of people of Mauritius actually belonged to India ». La fréquence et la réciprocité de ces visites officielles s'expliquent non seulement par la solidarité ethno-culturelle qui rapproche les leaders, mais aussi par l'importance stratégique que les gouvernements représentent l'un pour l'autre. Ainsi, lors de l'amputation de l'archipel des Chagos par la Grande-Bretagne et la création de la base militaire américaine sur l'île de Diego Garcia en 1965, l'Inde a vivement dénoncé la violation du territoire mauricien, dans le cadre du mouvement des non-alignés. En 1982, le Premier ministre de l'Inde a signé avec son homologue mauricien un communiqué rappelant les objectifs communs aux deux États, parmi lesquels figure la reconnaissance de la souveraineté de Maurice sur les îles Chagos et Diego Garcia. Indira Gandhi a également fait don d'un million de roupies indiennes aux Chagossiens déportés à Maurice, sous un prétexte humanitaire qui n'est pas sans lien avec la défense des intérêts géostratégiques de New Delhi dans la région.

L'Inde se positionne généralement en faveur de Maurice au cours des négociations de l'OMC, et soutient les projets mauriciens tels que l'organisation d'une conférence des 
leaders politiques d'origine indienne, parallèlement aux réunions de l'Assemblée générale des Nations unies ou des pays membres du Commonwealth. New Delhi et PortLouis sont également liées par un mécanisme de consultation en matière de politique étrangère et de défense, un dispositif de surveillance des eaux territoriales mauriciennes par les navires de guerre indiens, un traité bilatéral d'extradition, ainsi que de nombreux accords de coopération commerciale, financière ou culturelle. Les liens privilégiés entre l'Inde et l'île Maurice constituent dès lors un alibi permettant à New Delhi de justifier sa politique interventionniste dans l'océan Indien, et de satisfaire ainsi ses ambitions en tant que puissance régionale.

La diaspora indo-mauricienne contribue également au rayonnement de l'Inde dans l'Océan Indien en faisant la promotion active de son héritage culturel à l'échelle régionale avec l'appui du gouvernement mauricien. En organisant chaque année un festival de l'Inde à Maurice, les Indo-Mauriciens offrent une fenêtre sur l'Inde aux résidents de l'Océan indien qui n'ont pas les moyens de se rendre dans le souscontinent. Pour la plupart trilingues, maîtrisant le français, l'anglais et une langue indienne (bhojpuri, hindi, ourdou ou tamoul ${ }^{23}$ ), les Indo-Mauriciens servent notamment d'interprètes et de source d'inspiration pour leurs voisins Indo-Réunionnais. Ainsi, la GOPIO Mauritius est à l'origine de la sensibilisation des PIO réunionnais à leurs racines, longtemps refoulées par l'administration de la Métropole. Assimilés à la culture française, les Indo-Réunionnais ont perdu l'usage de leur langue ancestrale, ont francisé leur patronyme et se sont pour la plupart convertis au catholicisme. Contrairement aux archives du Mahatma Gandhi Institute de Maurice qui permettent d'identifier les premiers engagés indiens dans l'île, celles de la Réunion ont en partie brûlé, empêchant les Indo-Réunionnais de retracer leur généalogie. Ne pouvant justifier leur ascendance indienne, ces derniers éprouvent des difficultés à obtenir leur statut de PIO et à bénéficier des facilités mises à disposition par l'Inde. A ce jour, seuls une dizaine d'Indo-Réunionnais sont en possession de la PIO card, alors qu'ils constituent entre 25 et $30 \%$ de la population insulaire, forte de 750000 habitants.

Par ailleurs, le dynamisme culturel des Indo-Mauriciens a incité les PIO de la Réunion à se doter d'institutions diasporiques et à renouer, à leur tour, avec leur pays d'origine. Suite à la mobilisation active de Dhundev Bahadoor, une branche de la GOPIO a été récemment inaugurée à la Réunion et les associations socio-culturelles s'y sont multipliées, proposant des services de recherche généalogique, des voyages en Inde, des cours de langue, de danse ou encore des classes de yoga. Les projets de création d'un centre culturel indien, de reconstitution d'une " porte de l'immigration " à Saint-Denis illustrent le rôle de la diaspora indo-mauricienne dans la diffusion de l'indianité dans l'Océan Indien, mais aussi en Europe, à laquelle la Réunion est rattachée. Les Indo-Réunionnais prennent ainsi exemple sur la diaspora indomauricienne et se mobilisent en faveur d'un renouveau identitaire, qui remet en cause le mode d'intégration jacobin de la France, au profit d'un pluralisme culturel inspiré du sous-continent via l'île Maurice.

\section{La diaspora au service de la puissance et du pluralisme indien à l'échelle globale}

Au niveau international, New Delhi sollicite activement le soutien de la diaspora à la diplomatie indienne; elle a notamment obtenu l'appui de nombreuses associations 
diasporiques ou de chefs d'État d'origine indienne en faveur de la candidature de l'Inde à un siège permanent au Conseil de sécurité des Nations unies. Le gouvernement mauricien a été l'un des premiers à manifester son soutien à l'Inde à la tribune des Nations unies :

"We firmly believe that a greater commitment has to be demonstrated by every member of the Organization in order to reach an agreement on a reformed Security Council based on wider representation, transparency and equitable geographical distribution with the inclusion of developing countries from Africa, Asia and Latin America as Permanent Members. Here we should like to reiterate that India fully deserves to have that status. ${ }^{24}$

41 Alors que le diplomate d'origine indienne Shashi Tharoor présentait sa candidature au poste de Secrétaire général des Nations unies en 2006, le gouvernement indien s'est beaucoup investi dans la campagne, mobilisant les représentations indiennes à l'étranger, s'appuyant sur le réseau diasporique et les groupes de pression, notamment sur le très influent Indian Caucus (l'équivalent d'un groupe parlementaire) aux ÉtatsUnis (Therwath, 2006), pour rallier le maximum de votes au Conseil de sécurité.

Par ailleurs, les associations ethno-culturelles et les groupes de pression indiens de la diaspora font la promotion de la culture et des valeurs indiennes sur la scène internationale, se mobilisant en particulier pour obtenir la reconnaissance du hindi comme l'une des langues officielles aux Nations unies ${ }^{25}$. Porté par New Delhi, ce projet s'appuie largement sur la diaspora hindiphone du monde entier. Ainsi en 2005, le gouvernement indien s'associe sous forme de Joint Venture au gouvernement mauricien pour créer à l'île Maurice le "Secrétariat mondial du hindi ", avec pour mission de promouvoir la langue hindi à l'échelle globale. Lors de la huitième World Hindu Conference organisée à New York en juillet 2007 en coopération avec la Bharatiya Vidya Bhavan, le ministre des Affaires étrangères mauricien, Anand Sharma, s'est fait l'avocat du hindi comme langue internationale et langue officielle des Nations unies. Ce projet est relayé par des militants de la diaspora, qui font circuler des pétitions ou tentent de sensibiliser l'opinion publique en faveur de ce projet.

43 Toutefois, la promotion internationale du hindi ne fait pas l'unanimité au sein du réseau diasporique et suscite de vives résistances, notamment de la part des communautés linguistiques non-hindiphones d'origine indienne, qui y voient une stratégie du gouvernement central pour contourner, par l'échelle globale, le consensus national visant à protéger les langues régionales en Inde. Les membres des minorités linguistiques, en particulier les tamoulophones qui sont de fervents opposants à l' " hindisation » en Inde (Ramaswamy, 1997), profitent du mouvement en faveur de la reconnaissance du hindi comme langue officielle aux Nations unies pour demander à l'institution supra-nationale de faire respecter la diversité linguistique en Inde :

«What Tamil people fear (as well as some other non-Hindi peoples) is that recognizing Hindi as an official language of the United Nations would embolden Indian government to continue with more vigour Hindi imposition and language discrimination within India. (...) United Nations, if it ever were to seriously consider Hindi as an official language, should ask India to stop Hindi imposition and language discrimination.» (Chezhian, 2007)

Cette stratégie d'exportation des enjeux locaux sur la scène internationale est ainsi utilisée à la fois par le gouvernement indien pour consolider certains pans de l'héritage national, et par les États fédérés, en protestation contre les risques d'assimilation à la culture dominante. Tandis que le gouvernement central et les États hindiphones font la 
promotion du hindi, les autres États défendent leurs particularismes sur la scène internationale, en prenant appui sur la diversité des communautés diasporiques pour consolider leur place dans la mosaïque culturelle indienne. Le Tamil Nadu subventionne ainsi des cours de langue tamoule à l'étranger et co-organise de nombreuses conférences, conjointement avec les communautés diasporiques, sur le patrimoine culturel et linguistique tamoul. Cette politique s'inscrit dans le cadre d'un processus plus large de décentralisation des relations entre l'Inde, les États indiens et la diaspora plurielle. Depuis une dizaine d'années en effet, le gouvernement indien incite les États de l'Union à renforcer leurs liens directs avec leur diaspora régionale ${ }^{26}$. Le Kerala, le Gujerat, l'Andhra Pradesh, le Tamil Nadu ou encore le Bihar, principaux États d'émigration, ont ainsi adopté de nombreuses mesures destinées à leur diaspora respective, créant des institutions et des statuts spécifiques à l'usage des non-résidents (Non Resident Gujeratis, Non Resident Kerali, Non Resident Bihari ...), facilitant les flux d'investissements et encourageant les projets philanthropiques. La visite officielle du Premier ministre mauricien actuel au Bihar en 2008 s'inscrit dans cette stratégie d'instrumentalisation des connexions transnationales à des fins de développement local : Navin Ramgoolam a ainsi été invité par le chef du gouvernement du Bihar, Nitish Kumar, pour inaugurer une statue à l'effigie de son père, Seewoosagur Ramgoolam, aujourd'hui considéré comme le "père de la nation mauricienne ». En juillet 2008, le Festival de l'Inde à Maurice mettait le Bihar à l'honneur, et en 2009, ce sera au tour du Tamil Nadu. La célébration des liens historiques et ethniques entre le Bihar, l'État le moins développé de l'Inde, et l'île Maurice, considérée comme un "miracle économique » et un modèle de démocratie plurielle, permet de rehausser à la fois le prestige du Bihar en Inde et celui des Biharis en diaspora. A plus long terme, cette nouvelle politique diasporique cherche à attirer la bienveillance des 'successful Bihari' du monde entier, sous forme d'envois de fonds, d'investissements ou de dons caritatifs.

En faisant des Indo-Mauriciens les ambassadeurs ethno-culturels de l'Inde à Maurice, l'Inde se sert des PIO à des fins de développement local et national, mais aussi de rayonnement culturel et politique à l'échelle internationale. La diaspora constitue ainsi un instrument transnational de politique infra-nationale, sollicité par les autorités à la fois nationales et régionales de l'Inde, qui en font le terrain privilégié de promotion du pluralisme sur la scène transnationale.

\section{Conclusion}

La récente réhabilitation de la diaspora par New Delhi résulte non pas tant d'une solidarité ethnique par rapport aux émigrés indiens que d'enjeux économiques, stratégiques et culturels dans le cadre de la politique d'affirmation de l'Inde, aux échelles régionale et mondiale. Parallèlement, la mobilisation diasporique des PIO à l'île Maurice, mue par des intérêts à la fois locaux et transnationaux, a des répercussions bien au-delà de l'espace insulaire, venant notamment conforter la politique étrangère de New Delhi. Cette convergence des intérêts communautaires et étatiques s'inscrit dans le cadre d'un nouveau partenariat diaspora-État qui, loin de déstabiliser ce dernier, tend au contraire à le consolider tout en facilitant son intégration dans l'économie mondialisée. L'exemple de l'île Maurice développé dans cet article montre que la diaspora indienne constitue l'instrument privilégié du rayonnement culturel de l'Inde, et qu'elle agit également comme un pilier de la nouvelle Realpolitik de New Delhi 
sur la scène internationale, en faisant la promotion de l'Inde comme puissance militaire et économique, non seulement dans l'Océan indien, mais aussi dans le monde.

En ce sens, la diaspora se situe à l'interface des deux tendances observées aujourd'hui dans la sphère politique indienne: une approche multilatéraliste inscrite dans la continuité de la politique idéaliste incarnée par Nehru et Gandhi d'une part, d'autre part une approche unilatéraliste, plus réaliste et plus agressive, inspirée par le parti hindou du BJP (Jaffrelot, 2008). La combinaison de la diplomatie ethno-culturelle et de la politique économique de New Delhi à l'égard de la diaspora s'inscrit dans la quête de puissance de l'Inde sur la scène régionale et internationale, et se traduit, en Inde, par une décentralisation administrative et un renforcement du pluralisme culturel. En tant qu'ambassadeur informel des valeurs de l'Inde, la diaspora apparait dès lors comme une véritable courroie de transmission d'un modèle stato-national décentralisé et pluriel sur la scène internationale, en alternative au paradigme de l'État-nation centralisé et homogène. L'instrumentalisation de la diaspora par New Delhi dépasse ainsi le cadre de la diplomatie ethno-culturelle pour s'inscrire dans le champ de ce que Suzanne Nossel qualifie de Smart Power (2009), c'est-à-dire la combinaison des outils de coercition (militaires, économiques, politiques, juridiques) et de persuasion (diplomatie, assistance humanitaire, relations culturelles et économiques), ou encore l'association du soft power et du hard power (Nye, 2004 ; Nye, Amritage, 2007), au service des ambitions de l'Inde en tant que puissance émergente.

\section{BIBLIOGRAPHIE}

BENOIST Jean, « La diaspora indienne », Colloque : L'Inde grande puissance de l'Océan Indien, Publication du CHEAM, Le Centre des Hautes Études d'Aix-Marseille, 7-8 janvier 1988.

CARTER M., K. TORABULLY, Coolitude: an Anthology of the Indian Labour Diaspora, London, Anthem, 2002.

CHEZHIAN K., " Hindi in United Nations », Tamil Tribune, A monthly Magazine devoted to Tamil Issues, 17, 9, September 2007, disponible sur : http://www.geocities.com/tamiltribune/ 200709.html\#one1, consulté le 25 avril 2009.

COHEN Robin, Global Diasporas: an Introduction, Seattle, University of Washington Press, 1997.

COHEN Stephen, India: Emerging Power, Washington, Brookings Institutions, 2001.

DUFOIX Stéphane, « Généalogie d'un lieu commun, 'diaspora' et sciences sociales », Groupe d'Etude et d'Observation de la Démocratie-GEODE, séminaire d'histoire sociale de l'immigration de l'ENS, 2002, disponible sur : http://barthes.ens.fr/clio/revues/AHI/articles/preprints/ duf.html\#fn1

DUFOIX Stéphane, L'objet diaspora en questions', Cultures et Conflits, 33-34, printemps-été 1999, p. 147-163.

DUFOIX Stéphane, Les diasporas, Paris, Presses universitaires de France, 2003. 
GANDHI Rajiv, «Inaugural Address to the Conference of Education Ministers of State and Union Territories, New Delhi, 29 August 1985 », in Rajiv GANDHI, Selected Speeches and Writings, Publications Division, Ministry of Information and Broadcasting, Government of India, New Delhi, 1987.

GANGULY Sumit (ed), India as an Emerging Power, London, Franck Cass, 2003.

GUILMOTO Christophe, « Le cycle migratoire tamoul, 1830-1950 », Revue Européenne des Migrations Internationales, 7, 1, 1991, p. 123-149, disponible sur : http://www.persee.fr/web/revues/home/ prescript/article/remi_0765-0752_1991_num_7_1_1280

HELLY Denise, 'Diaspora : un enjeu politique, un symbole, un concept ?', Espace populations sociétés, 1, 2006, p. 17-31.

HOOKOOMSING VINESH Y., "Chota Bharat, Mauritius: from Myth to Reality », in Bikhu PAREKH, Gurharpal SINGH, VERTOVEC Steven, Culture and Economy in the Indian Diaspora, London, Routledge, 2003, p. 13-32.

Indian Centenary Book, Published on the occasion of the Second World Hindi Convention, Moka, MGI Institute Press, 1976 ( $1^{\text {st }}$ edition, 1936).

JAFFRELOT Christophe, New Delhi et le monde : une puissance émergente entre Real Politik et Soft Power, Paris, Editions Autrement, 2008.

LALL Marie Carine, India's Missed Opportunity: India's Relationship with the Non Resident Indians, London, Ashgate, 2001.

LANDY Frédéric, « Terre des ancêtres et territoire de diaspora. Le cas des Sud-Africains d'origine indienne », in GERVAIS-LAMBONY Philippe, LANDY Frédéric, OLDFIELD Sophie (dir), Espaces arcen-ciel. Identité et territoire en Afrique du Sud et en Inde, Paris, Géotropiques / Karthala/IFAS, 2003, p. 187-206.

Ministry of Overseas Indian Affairs, Annual Report, 2004-2005.

Ministry of External Affairs, The Report of the High Level Committee on Indian Diaspora, Ministry of External Affairs, New Delhi, 2002, disponible sur : http://www.indiandiaspora.nic.in/ contents.htm

NAYAR Baldev Raj, Thazha Varkey PAUL, India in the World Order: Searching for Major Power Status, Cambridge, Cambridge University Press, 2003.

NOSSEL Suzanne, « Smart Power », Foreign Affairs, March-April 2004, disponible sur : http:// www.foreignaffairs.com/articles/59716/suzanne-nossel/smart-power, consulté le 10 mars 2009. NYE Joseph S., Soft Power, the Means to Success, New York, Public Affairs, 2004.

NYE Joseph S., Richard L. AMRITAGE, Report of the CSIS Commission on Smart Power, 'A smarter, more secure America', Washington, CSIS, 2007, disponible sur : http://www.csis.org/media/csis/ pubs/071106_csissmartpowerreport.pdf, consulté le 10 mars 2009.

PAREKH Bikhu, « The Indian Diaspora », in J. K. MOTWANI, M. GOSINE, J. BAROT-MOTWANI (ed), The Global Diaspora: Yesterday, Today and Tomorrow, New York, Global Organization of People of Indian Origin, 1993, p. 8-11.

PERCOT Marie, «Les infirmières indiennes émigrées dans le Golfe : de l'opportunité à la stratégie » Revue Européenne des Migrations Internationales, 21, 1, 2005, p. 29-54

RAMASWAMY Sumathi, Passions of the Tongue: Language Devotion in Tamil India, 1891-1970, Berkeley, London, University of California Press, 1997. 
THERWATH Ingrid, « Diaspora : la voix de Delhi aux États-Unis », Alternatives Internationales, 32, septembre 2006.

TINKER Hugh, A New System of Slavery: the Export of Indian Labour Overseas, 1830-1920, London, Oxford University Press, 1974.

VENIER Philippe, Travail dans le Golfe Persique et développement au Kérala : les migrants internationaux, des acteurs au cour des enjeux sociaux et territoriaux, Thèse de doctorat (géographie), Université de Poitiers, 2003.

World Bank, Migration and Remittances Factbook 2008, disponible sur : http://web.worldbank.org/ WBSITE/EXTERNAL/NEWS/0,,contentMDK:21692926 pagePK:64257043 piPK:437376 theSitePK: 4607,00.html, consulté le 12 mai 2009.

ZINS Max Jean, « L'Inde et l'Afrique : le non-alignement au service des intérêts nationaux », Politique Africaine, 10, 1983, p. 39-53.

\section{NOTES}

1. Dans la théorie des Relations internationales, la notion de 'soft power' traduit la capacité d'une entité socio-politique à influencer indirectement le comportement d'une autre entité sociopolitique par le biais de moyens culturels ou idéologiques, en contraste avec le 'hard power', c'està-dire l'usage de moyens de coercition tels que la pression politique ou économique, ou encore l'usage de la force militaire. Voir NYE, 1990, 2004.

2. La catégorie fiscale NRI (Non Resident Indians) est apparue en 1973 dans l'India Foreign Exchange Regulation Act (FERA). Les NRI sont des ressortissants indiens résidant et travaillant à l'étranger pour une durée supérieure à 180 jours par an. Aujourd'hui, les NRI peuvent obtenir la 'carte PIO', ou le statut d'OCI (Overseas Citizen of India) qui leur donne droit à quasi tous les droits civiques des ressortissants indiens, à l'exception du droit de vote et de l'acquisition de terres agricoles en Inde. La majorité des NRI, qui ont migré à partir de la seconde moitié du $\mathrm{XX}^{\mathrm{e}}$ siècle, sont aujourd'hui établis soit dans les pays du Moyen-Orient, soit dans les pays développés, en Amérique du Nord, en Europe et en Australie.

3. L'acronyme PIO (Person of Indian Origin) se réfère aux ressortissants étrangers ne provenant ni du Pakistan, ni du Bengladesh, qui ont été citoyens indiens dans le passé, ou descendants de citoyens indiens jusqu'à la quatrième génération, mais qui ont renoncé à leur nationalité pour adopter celle de leur pays d'installation (LALL, 2001, p.180-181). La plupart des PIO ont quitté l'Inde au cours de la vague migratoire du XIX ${ }^{\mathrm{e}}$ siècle, dans le cadre des systèmes de l'engagisme, du kangani ou du maystri, et se sont sédentarisés dans les plantations coloniales, en Afrique, en Asie, en Amérique Centrale et dans les îles de l'océan Pacifique, de l'Océan Indien et des Caraïbes.

4. Le terme 'diaspora' fait l'objet de nombreuses interprétations en sciences humaines, et son usage est aujourd'hui passé dans le vocabulaire journalistique et politique, ainsi que dans le langage courant. Par 'diaspora', on entend généralement toute communauté issue de l'immigration, en liens - réels ou imaginaires - avec son pays d'origine, et animée par le 'mythe du retour'. A propos de l'étymologie du terme 'diaspora' et de son évolution sémantique, voir Dufoix, 1999, 2002, 2003. Pour une tentative de classification des différentes diasporas, voir COHEN, 1997 ; Pour une étude de la valeur à la fois normative et performative de la catégorie de diaspora, voir HELLY, 2006.

5. US Census Bureau, Selected Population Profile, Asian Indian alone or in any combination, 2007, disponible sur: http://factfinder.census.gov. En 2007, les Américains d'origine indienne composent $0,9 \%$ de la population totale.

6. Ministry of External Affairs, 2002, p. 25. 
7. Statistique Canada, Recensement, 2006.

8. Le terme « coolie » se réfère aux travailleurs indiens et chinois engagés dans les plantations et les grands travaux de construction, au lendemain de l'abolition de l'esclavage. Les coolies travaillaient pour des rémunérations minimes, dans des conditions particulièrement difficiles. Le terme, d'origine tamoule, s'est progressivement chargé d'une connotation péjorative et d'une valeur à la fois normative et performative, visant à la fois à désigner les ouvriers, mais également à justifier les traitements infligés afin de les maintenir dans une situation de précarité.

9. Hazareesingh, 1936, p. 8.

10. D'après une tradition établie depuis la fin du XIX ${ }^{\mathrm{e}}$ siècle, chaque année, une journée de septembre était consacrée à des épreuves humiliantes mettant en scène des Indo-Mauriciens, destinés à susciter les moqueries des spectateurs. La pratique la plus connue et la plus dégradante consistait à faire grimper un malheureux à un mât de cocagne pour exciter la foule. $\mathrm{A}$ la suite d'une campagne contre les courses malabars initiée par Basdeo Bissoondoyal, les travailleurs indo-mauriciens ont boycotté l'évènement, qui a finalement été interdit en 1947.

11. Depuis la lutte en faveur de l'Indépendance de l'île jusqu'à nos jours, la prépondérance des hindous originaires du Nord de l'Inde au gouvernement constitue un trait majeur de la vie politique mauricienne.

12. Current Affairs Publication, Nehru on Africa, New Delhi, 1954, cité in ZINS, 1983, p..

13. Les travailleurs indiens du Moyen-Orient font aujourd'hui de l'Inde le premier bénéficiaire de fonds privés au monde (World Bank, 2008).

14. En 2000, le gouvernement indien met en place un comité (High Level Committee on the Indian Diaspora) chargé de recenser pour la première fois les membres de la diaspora, et d'émettre des recommandations en vue d'améliorer les relations entre l'Inde et les émigrés. Voir le rapport en ligne, disponible sur : http://www.indiandiaspora.nic.in/contents.htm

15. La recherche généalogique des ancêtres en Inde, proposée par le Mahatma Gandhi Institute (île Maurice), vise avant tout à rapprocher les Indo-Mauriciens de leur pays d'origine et à resserrer les liens émotionnels, mais aussi politiques et économiques, entre les deux pays.

16. Dans la numérologie hindoue, 108 représente le nombre de noms donnés à Shiva, ou Nataraj, le roi de la danse, à qui on attribue généralement 108 karana (mouvements), qui composent le tandava, une danse cosmique allégorique des cinq principales manifestations de l'énergie éternelle, que sont la création, la destruction, la préservation, la salvation et l'illusion. Les mala (chapelets de prière) sont également composés de 108 perles. Suite à la construction de la statue géante de Shiva, une effigie de Ganesh, de taille identique, a été construite à l'occasion du festival Ganesh Chaturthi, en septembre 2007, par la communauté marathie de Bois Chéri, une ville voisine de Grand Bassin. Les différentes communautés indo-mauriciennes se livrent ainsi une véritable compétition en vue d'accroître leur prestige et leur statut dans l'île, en valorisant l'héritage culturel ou spirituel indien.

17. Tandis que l'usage initial qualifie de "créole» toute personne née dans les colonies d'outremer, ce terme a par la suite acquis une connotation raciale, désignant dans un premier temps les 'Blancs' des colonies, avant d'être étendus aux métis et mulâtres. A l'île Maurice, ce terme revêt une signification particulière: il se réfère aux personnes d'ascendance africaine, qu'elles soient métissées ou non.

18. La famille Ramgoolam a quitté le Bihar pour travailler dans les plantations mauriciennes à la fin du XIX ${ }^{\mathrm{e}}$ siècle. Après avoir mené la lutte en faveur de l'Indépendance, Seewoosagur Ramgoolam est devenu, en 1968, le premier chef de gouvernement de l'État insulaire.

19. Le concept de " coolitude ", poétisé par Khal Torabully, vise à célébrer la diversité culturelle de l'ère contemporaine, définie comme transculturelle, voir Carter, Torabully, 2002.

20. Alors que les descendants des esclaves s'enlisent dans le «malaise Créole » à Maurice, le recours au mythe de la communauté modèle permet au gouvernement mauricien de se 
désengager vis-à-vis de la population créole, et de valoriser au contraire la trajectoire des descendants des engagés indiens.

21. Les 14 célèbres marches de l'Aapravasi Ghat ont dernièrement été foulées par le Premier ministre indien Manmohan Singh le $1^{\text {er }}$ avril 2005, le Président Abdul Kalam le 12 mars 2006, le ministre des Affaires des Indiens d'outremer Valayar Ravi le 22 novembre 2006, le chef du gouvernement du Bihar Nitish Kumar en juillet 2007, ainsi que par de nombreux ministres d'État, leaders religieux et artistes indiens.

22. Il convient de rappeler l'existence, au sein de la diaspora, de mouvements internes hostiles aux intérêts de l'État indien, à l'instar de la mouvance pro-khalistanie qui, au cours des années 1980-1990, caressait le projet de création d'un État autonome pour les sikhs. Au cours des années 1970, de nombreuses associations diasporiques indiennes progressistes et libérales se sont également opposées au gouvernement indien, contestant l'État d'urgence décrété par Indira Gandhi et la suppression des libertés qui s'en est suivie. Parallèlement, l'interdiction en Inde du mouvement fondamentaliste hindou Rashtriya Swayamsevak Sangh (RSS), soupçonné d'être lié à l'assassinat du Mahatma Gandhi, a aussi stimulé l'émergence d'associations diasporiques hostiles à l'appareil d'État indien. Loin de s'exprimer d'une seule voix, la diaspora indienne est composée d'une pluralité d'acteurs qui soutiennent ou s'opposent au gouvernement indien selon leurs intérêts propres, qu'ils soient communautaires ou individuels.

23. Pour la plupart originaires du Nord de l'Inde, les Indo-Mauriciens parlent encore aujourd'hui le bhojpuri, dialecte dérivé du hindi de la région qui correspond actuellement à l'État du Bihar.

24. Mauritian Minister for Foreign Affairs, Jayen Cuttaree, 59ème Assemblée Générale des Nations Unies (28/09/2004). Voir le site http://www.meaindia.nic.in/foreignrelation/ Mauritius.htm

25. Le hindi est la langue la plus parlée en Inde et la seconde langue la plus parlée au monde après le mandarin, mais à ce jour elle ne figure pas parmi les six langues officielles des Nations unies.

26. Les États tels que le Tamil Nadu ou l'Andhra Pradesh entretiennent des relations privilégiées avec les Tamouls et les Télougous d'outremer depuis les années 1970, à travers la promotion culturelle de leur identité régionale à l'étranger. Depuis les années 2000, le gouvernement indien encourage les États à institutionnaliser ces relations États indiens-diaspora, sur le modèle de la relation État central-diaspora.

\section{RÉSUMÉS}

Les Persons of Indian Origin (PIO) ont depuis le début du XX $\mathrm{XX}^{\mathrm{e}}$ siècle servi de levier à l'intelligentsia indienne pour rehausser l'image du pays et contribuer à son rayonnement culturel sur la scène internationale, en tant qu'ambassadeurs culturels de l'Inde dans leur pays de résidence. Aujourd'hui, les nouveaux liens établis entre New Delhi et les Non Resident Indians (NRI) dépassent largement le cadre de la diplomatie culturelle, et leur mobilisation économique ou politique en faveur du traité nucléaire contribuent à l'affirmation de la puissance de l'Inde. Une analyse de la mobilisation des Indo-Mauriciens permet de mettre en lumière le double rôle du facteur diasporique dans l'émergence de l'Inde comme grande puissance régionale et internationale, en tant qu'instrument à la fois du Soft Power et de la Realpolitik de New Delhi. 
People of Indian Origin (PIOs) have, since the beginning of the $\mathrm{XX}^{\text {th }}$ century, been used by the Indian intelligentsia to promote the image of the sub-continent and diffuse India's cultural values on the international stage, as cultural ambassadors of India in their host countries. Today, the new partnership between New Delhi and the Non Resident Indians (NRIs) go beyond India's cultural diplomacy, and the support of the Diaspora in favour of the nuclear deal plays a strategic part in the affirmation of India as an emerging power. An analysis of the mobilisation of IndoMauritians in line with the interests and values of India illustrates the double role of the diasporic factor in the emergence of India as a regional and global power, as a tool of New Delhi's soft power and Realpolitik.

\section{INDEX}

Keywords : Indian diaspora, cultural diplomacy, India, Mauritius Island, smart power

Mots-clés : diaspora indienne, diplomatie culturelle, Inde, Ile Maurice

\section{AUTEUR}

\section{ANOUCK CARSIGNOL-SINGH}

Anouck Carsignol-Singh (anouck.carsignol@graduateinstitute.ch) est Docteur en Relations Internationales à l'Institut de Hautes Etudes Internationales et du Développement (IHEID) à Genève, $\mathrm{CH}$. Elle a notamment publié :

- 'From Coolies to Cultural Elites: Indo-Mauritians, Transnational Diasporic Organizations, and the Shaping of Mauritian Nation-State', in MAHASE R., TEELUCKSINGH J., Indentureship to Globalization: New Perspectives on the Indian Diaspora, Lanham, Lexington Press, à paraître, 2009 -'Les Indo-Mauriciens en France: au carrefour des diasporas', Hommes et Migrations, numéro spécial « Les Indiens en France », Paris, Adri, 2007, p.120-128. 